\title{
Neural systems underlying affective disorders
}

\section{Simon Surguladze, Paul Keedwell \& Mary Phillips}

\begin{abstract}
Three main approaches are used to explore the neural correlates of mood disorder: neuropsychological studies, neuroimaging studies and post-mortem investigations. Lesion studies implicate disturbances in the frontal lobe, basal ganglia, striatum and anterior temporal cortex. Early neurocognitive and neuropathological investigations led to a 'hypofrontality' hypothesis of unipolar and bipolar depression, but functional neuroimaging has revealed a more complex picture. Thus, increased metabolism may occur in the subgenual anterior cingulate gyrus in resting-state studies of depression and sad-mood induction. Antidepressants may reduce this activity. Amygdala hyperactivation also is associated with affective disorders. Task-related studies reveal abnormal biases in memory, the experience of pleasure and the perception of emotional facial expressions. There is still little clarity whether the abnormalities in brain activation represent state or trait characteristics of affective disorders.
\end{abstract}

In this article we focus on the abnormalities in brain function that are associated with mood change in the affective disorders, including major depressive disorder and bipolar affective disorder. Despite the fact that these conditions are common and cause considerable disability, little is known about the functional circuits in the brain that mediate their symptoms. A greater knowledge of these processes will provide a rigorous test of our current diagnostic boundaries. In combination with neurochemical research, an improved knowledge of functional neuroanatomy will have implications for prognosis and the development of new treatments.

\section{Neuropsychological findings Lesion-deficit studies}

Lesion-deficit studies of neurologically depressed subjects have focused on three main clinical groups: those with discrete lesions, those with known neurochemical deficits such as Parkinson's disease, and those diseases with generalised or randomly distributed pathology such as Alzheimer's disease or multiple sclerosis.

In one of the first documented cases of emotional disorder due to a brain lesion, Phineas Gage famously suffered from accidental damage of the frontal lobe in 1848. The iron bar that forced its way through his cranium caused lesions in the left and right ventromedial regions of the prefrontal cortex (as reconstructed by Damasio et al, 1994). Mr Gage, who was an exemplary citizen before the accident, afterwards became a completely different person - impatient, rude and prone to outbursts of anger and rage. Blumer \& Benson (1975) proposed that personality changes after frontal lobe damage could be classified under 'pseudopsychopathy' or 'pseudodepression'. Pseudodepression was more frequently associated with lesions of the left frontal lobe and was characterised by apathy, loss of initiative, low mood and reduced sexual interest. Several lesion studies have demonstrated that damage to left frontal areas contributed specifically to depressive syndromes, whereas the consequences of right prefrontal lobe damage are less clear and may be associated with pseudopsychopathic behaviour or mania. Although the lateralisation effect has not been found in all studies, the association of mood disturbance with prefrontal lesions is widely recognised.

Studies that reviewed the structural brain scans of stroke patients found an association between subsequent mood changes and infarctions of the

Simon Surguladze is a research worker in the Section of Neuroscience and Emotion, King's College London, based at the Institute of Psychiatry (De Crespigny Park, Denmark Hill, London SE5 8AF, UK). Paul Keedwell is an honorary clinical research worker and specialist registrar at the Mood Disorder Clinic of the Maudsley Hospital, London. Mary Phillips is Head of the Section of Neuroscience and Emotion and professor and honorary consultant psychiatrist in the Division of Psychological Medicine, Institute of Psychiatry and GKT School of Medicine. The Section's research programme includes studies of neurobiological mechanisms underlying mood dysregulation in patients with affective disorders, schizophrenia, depersonalisation and obsessive-compulsive disorder. 
frontal lobe and basal ganglia, particularly the head of the caudate. Similar studies of trauma cases and tumour patients have suggested that dorsolateral rather than ventral frontal lobe lesions are important for depressive mood change, but these are heterogeneous conditions and there are inconsistent findings. The importance of temporal lobe pathology in the genesis of organic depression is suggested by several studies demonstrating a link between depression and lateralised temporal lobe epilepsy, the presence of temporal lobe plaques in multiple sclerosis and the extent of temporal involvement in Alzheimer's disease sufferers. Pathology in the corpus striatum, an area believed to be important for regulating the pleasure response (among other functions), is also implicated in these studies.

The nigrostriatal pathway is affected in Parkinson's disease, a condition known to have a high association with depression. There are indications from post-mortem studies that selective cell loss in the ventral tegmentum (an essential component of the dopamine-mediated 'reward system' - see below) must also occur for prominent mood symptoms to be present.

The findings from lesion studies are consistent with disruption in two known anatomical pathways, as illustrated by Mayberg (2000): the orbital-frontal-striatal-thalamic circuit and the basotemporal-limbic circuit, which links the frontal cortex to the anterior temporal cortex.

\section{Cognitive tasks}

Cognitive tasks have been developed that are designed to detect abnormalities in functionally distinct areas of the brain. The rationale behind their use in mood disorders is to investigate certain areas of interest, often guided by lesion studies or neuropathological findings. The aims of the studies fall into two main camps: those that are hoping to demonstrate functional abnormalities in brain areas thought to be important in the mediation of affective psychopathology, and those that wish to demonstrate cognitive disability consequent on affective disorder.

Neuropsychological paradigms have been largely led by lesions studies. It is not surprising, therefore, that most of the literature focuses on tests of frontal lobe function in major depressive disorder and bipolar affective disorder.

Deficits in executive function have been demonstrated in major depressive and depressed bipolar affective disorders. However, some researchers have questioned whether these deficits are discrete, suggesting that they are part of a more generalised, diffused impairment (for a review see
Veiel, 1997), implicating frontal executive, attentional and memory systems. Declarative memory (requiring effortful recall) is known to be more impaired than implicit memory (Danion et al, 1991; Bazin et al, 1994).

Mood-congruent memory bias is a consistent finding in both major depressive disorder and depressed bipolar affective disorder. The bias towards recall of subjectively unpleasant over pleasant material has been demonstrated in both explicit and implicit recall (priming) experiments (Watkins et al, 1996).

Whether the cognitive deficits or biases described thus far in people with either of these two disorders represent trait or state effects is an important issue. Cognitive performance in general has been shown to improve on the symptomatic recovery from episodes of major depressive disorder. However, the story may be different for bipolar affective disorder: persistent problems with spatial working memory, setshifting and sustaining attention have been reported, and the severity of various cognitive deficits has been shown to correlate with longer illness duration and the number of manic and depressed episodes (Cavanagh et al, 2002; Clark et $a l, 2002)$. Those with a history of psychotic symptoms may perform no better than people with schizophrenia in remission. These findings have challenged the established view that bipolar disorder with psychotic symptoms is associated with a better prognosis, in terms of neuropsychiatric disability, than psychotic illnesses such as schizophrenia.

However, the finding that residual affective symptoms are commonly found in people with socalled 'remitted' bipolar affective disorder is of crucial importance. Some authors have shown that most cognitive deficits disappear when residual depressive symptoms are controlled for (Ferrier et al, 1999; Clark et al, 2002).

In summary, the neurocognitive abnormalities found in major depressive disorder and bipolar affective disorder probably represent state effects of the illness process, particularly the depressive illness. There is evidence of abnormal frontal lobe functioning in both disorders. However, they occur in the context of wider cognitive disability.

Some neuropsychological approaches have focused more on perceptual processes and, in particular, the effect of depression on the recognition or identification of non-verbal social cues. For example, people who are currently depressed may be particularly poor at emotion identification (Cooley \& Nowicki, 1989). Such difficulties could lead to problems in social relationships, which, in turn, could aggravate the depressive state. 
Among the processes involved in social cognition, the ability to recognise emotional facial expressions is crucial. Rubinow \& Post (1992) found that patients in an episode of depression were significantly poorer than normal controls in the processing of facial but not verbal emotional expressions. This finding was confirmed in a number of other studies.

However, Gur et al (1992) suggested that people in a depressed state are negatively biased, rather than generally impaired, in their perception of emotions in others. During a depressive episode people have been shown to misinterpret neutral faces as sad and happy faces as neutral. This tendency discriminated them from the healthy controls. Matthews \& Antes (1992) and Hale (1998) have also demonstrated a negative bias in the identification of different emotional states. Suslow et al (2001) found that the recognition of faces expressing negative emotions was not 'enhanced' in people during episodes of mild depression or dysthymia, but they detected an impairment in the processing of positive facial expressions.

Importantly, as for the cognitive deficits, the emotion recognition deficits identified thus far may recover on resolution of the affective symptoms. They have been found to subside with remission (Mikhailova et al, 1996). The deficits may reflect abnormal activity in dedicated 'facial neurons', which have been identified in animal experiments. The facial neurons are believed to be located in the amygdala, inferior temporal cortex and orbitofrontal cortex in animals (Perrett et al, 1982). As we shall see in the following sections, neuropathological and neuroimaging studies support the idea that depression is associated with abnormalities in the orbitofrontal cortex and the amygdala.

\section{Neuropathological findings}

Post-mortem studies can reveal important information about disease processes. The brains of deceased patients with a history of mood disorder can be compared with brains of controls without or with other confounding brain disease. The main failing of neuropathological investigation is that it is not always possible to control for independent medication effects. Also, dead tissue can give us only a limited insight into the dynamic disease state. However, recent advances in neuroanatomical techniques have facilitated more detailed post-mortem investigations. A thorough examination of cortical and subcortical tissues is possible, providing information about the number, size, shape and distribution of cells. Studies conducted so far suggest that the histopathological picture in the affective disorders with psychotic symptoms is distinct from the picture seen in psychoses such as schizophrenia.

Reduced numbers of glial cells and a reduced neuronal cell density have been found at postmortem examination in the dorsal anterolateral prefrontal cortex, subgenual anterior cingulate gyrus (below the genu of the corpus callosum), orbitofrontal cortex (near the olfactory tracts) and ventrolateral prefrontal cortex in individuals who had either major depressive disorder or bipolar affective disorder (Ongur et al, 1998; Cotter et al, 2001; Rajkowska et al, 2001). Glial cells provide many essential services to the neuron, including physical support, nourishment and the removal of dead tissue. They occur in much greater numbers than neuronal cells, so a reduction in the number of glia will have a direct effect on neuronal density. However, the actual number of neurons is typically unchanged in those with a history of mood disorder. The disease process underlying mood disorder is therefore unlikely to be neurodegenerative. Drevets (2000) has suggested that the 'excitotoxic' effects of elevated levels of glutamate are responsible for the observed grey matter changes.

Reduced glial cell and neuronal density may also occur in deeper brain structures. Baumann et al (1999) demonstrated smaller grey matter volumes in the caudate and nucleus accumbens in patients with major depressive disorder or bipolar affective disorder when compared with controls. Bowley et al (2002) uncovered a reduction of glial cell counts, glial density and glia-toneuron ratio in the amygdala of patients with major depressive disorder (most of whom did not receive valproate or lithium), but not of those with bipolar affective disorder (most of whom had been treated with valproate or lithium). The authors noted an effect of lithium or valproate treatment: the bipolar affective disorder patients who did not receive lithium or valproate had a significant glial reduction similar to that seen in patients with major depressive disorder. So, mood stabilisers may be neuroprotective.

Mood disorders were traditionally regarded as having a good prognosis compared with psychotic disorders. The reduced glial cell numbers found in the limbic-cortical circuits in those with a history of affective illness might lead one to challenge this view. One might conclude that having a mood disorder will lead to persistent deficits in brain function. However, some neuropathological studies have suggested a neuroprotective effect of psychotropic medication, which 
lends support for therapeutic optimism (Manji et al, 2000). Furthermore, the findings of some neuroimaging studies suggest that changes in brain structure may be entirely reversible on recovery from an episode, as we shall see.

\section{Neuroimaging}

\section{Structural}

Structural magnetic resonance imaging (MRI) techniques provide us with an unprecedented possibility to measure the volume of various brain structures in the living patient and to corroborate these findings with the neuropathological data. Employing a strong magnetic field, rather than radiation, they give superior spatial resolution than do older X-ray methods such as computerised tomography.

In keeping with the neuropathological data, structural MRI has demonstrated reduced left subgenual anterior cingulate cortex volumes in patients with familial major depressive disorder or bipolar affective disorder (Drevets et al, 1997) and reduced caudate, amygdala and putamen volumes in major depressive disorder (Beyer \& Krishnan, 2002). Findings of hippocampal volume reduction have also been reported in major depressive disorder, with a postulated mechanism of glucocorticoid neurotoxicity (cortisol levels are often high in major depressive disorder). However, this hippocampal 'shrinkage' may be reversed by antidepressant treatment: research using rat brains shows that antidepressants stimulate neurogenesis in the hippocampus. The long-term use of lithium has been associated with an increase in the volume of the subgenual cingulate gyrus (Manji et al, 2000), further evidence of its neuroprotective effect.

Some investigators have found structural abnormalities on MRI that may be specifically associated with bipolar affective disorder. Thus, enlargement of the third ventricles (thought to reflect reduced thalamic and hypothalamic volumes) and amygdala abnormalities (mostly enlargement) are consistently shown. However, it is difficult to marry these findings with findings from post-mortem studies (above) that showed reduced grey matter volumes in the amygdala in unmedicated bipolar affective disorder, consistent with findings in major depressive disorder. Lithium-induced neurogenesis may contribute to the discrepant findings.

Some studies have demonstrated more hyperintense lesions (reflecting an increased water density) in the white matter of patients with bipolar affective disorder than in controls or patients with major depressive disorder. The proposed clinical implication of these findings is that connections between mood-regulating regions may be disrupted.

More work needs to be done in the area of structural imaging. Not only are the findings inconsistent, but it is unclear whether any of these structural changes represent state or trait effects. Also, they have not been clearly related to manic or depressive phases, and the effect of medication is difficult to exclude. Examining medicationnaïve patients with index episodes and studying the 'high-risk' offspring of patients with bipolar disorder might help to resolve these issues.

\section{Functional neuroimaging}

Advances in neuroimaging techniques have made it possible to examine changes in the function of the brain associated with cognitive processes. Two main methods have been used: functional MRI (fMRI) and positron emission tomography (PET). The former uses the blood oxygen level dependent (BOLD) response as an analogue of neuronal activity. Recent work (Logothetis et al, 2001) suggests that there is a close spatial and temporal relationship between increases in local field potentials created by cortical neuronal activation and increases in the flow of oxygenated blood to these activated areas. Oxygenated blood is more paramagnetic than deoxygenated blood and this difference is what fMRI is able to gauge. PET, on the other hand, indirectly measures neuronal activation by using radiotracers. These can detect the rate of neuronal glucose metabolism (by using fluorin-18) or regional cerebral blood flow (using oxygen-15). With PET it is also possible to measure receptor density by tracing the isotopes selectively binding to, for example, dopamine, serotonin and opiate or benzodiazepine receptors. Functional MRI has better temporal and spatial resolution than PET. Changes in the BOLD response can be detected every second using fMRI, whereas the temporal resolution of PET is in the order of minutes. Hence, fMRI is arguably more useful in a task- or stimulus-related study when the task is brief or rapidly repeated (e.g. in an event-related study). Functional MRI allows for paradigms in which the 'on' condition is rapidly alternated with the 'off' condition (an average of the responses across the two conditions is then analysed). This can help to control for independent stimuli, metabolic and drug effects, and individual differences in response.

However, on the plus side for PET, the link between metabolism and neural activity is well established. The meaning of the BOLD response 
is more hotly debated. For example, Attwell \& Iadecola (2002) argued that the haemodynamic response is determined by postsynaptic currents (i.e. neurotransmitter-related signalling) rather than by presynaptic energy consumption. Subsequently, this should be taken into account when interpreting data on imaging of psychiatric disorders, which are characterised by an abnormal metabolism of neurotransmitters.

Analysis of data from both types of imaging is difficult and complex. The voxel-based method is widely used. The brain is divided into thousands of voxels (the three-dimensional equivalent of the pixel). Every brain has a slightly different size and morphology, so data must be transposed into standard space using an agreed template of brain anatomy. Useful conclusions cannot be drawn from the analysis of a single brain. It is assumed that anatomical variations will average out over a large number of subjects. The template that is used by most researchers is the Talairach map - a detailed plan of brain anatomy that is used as the benchmark (Talairach \& Tournoux, 1988). The ultimate reliability of the spatial resolution will depend on the accuracy of the transformation and the validity of the benchmark. The greater the number of brains examined and the greater the number of studies carried out, the more confident we can be of the validity of their findings (assuming that they all demonstrate consistent results).

\section{Resting-state studies}

The results of early resting-state PET studies (e.g. Baxter et al, 1985) seemed to be consistent with the neuropathological findings of reduced neuronal density in the prefrontal cortex in bipolar affective disorder and major depressive disorder. They demonstrated reduced activation of parts of the prefrontal cortex in depressed patients with either disorder compared with healthy controls.

Controlling for structural changes in brain regions helps to clarify the results of functional neuroimaging studies. After correcting for reduced grey matter volume, more recent studies have demonstrated an increase in metabolic activity in the subgenual prefrontal area as measured by PET (e.g. Drevets et al, 1997; Mayberg et al, 2000). Furthermore, when examining the neural correlates of treatment with the antidepressant fluoxetine (Mayberg et al, 1999), increased restingstate activity in the subgenual prefrontal cortex of patients with major depressive disorder subsided with clinical improvement. Patients with persisting symptoms continued to have increased metabolism in this area. Further corroboration has been provided by the observation of increased activity in the subgenual prefrontal cortex of healthy volunteers during experimentally induced sadness. It has been suggested that this is an analogue of depressive brain activity. Other studies have shown increased activity in the medial prefrontal cortex of healthy subjects during sad-mood induction (Pardo et al, 1993; Northoff et al, 1999), although not specifically in the subgenual area. Reduced activity has been demonstrated in lateral prefrontal regions (Mayberg et al, 1999), and medial and lateral prefrontal activity may be dissociated for negative and positive moods respectively (Northoff et al, 1999). Although experimentally induced transient sadness is a very different experience from clinical depression, these studies help to map the functional networks involved in affective states.

Increased resting-state amygdala activity has been demonstrated in major depressive disorder and bipolar affective disorder but not in psychotic bipolar affective disorder (Drevets et al, 1992, 1995). Importantly, amygdala metabolism has been shown to correlate positively with depression severity (Abercrombie et al, 1998) and it appears to decrease following effective treatment.

There are complex relationships between the severity of depression and the cerebral blood flow in posterior orbital, left ventrolateral prefrontal cortex and anterior insula areas. Although the metabolism in these areas seems to be greater in patients with major depressive disorder than in the normal controls, neural activity correlates inversely with depression ratings. Drevets (2000) has argued that elevated activity in these areas is not essential for the production of depressive symptoms, but that it reflects endogenous attempts to 'attenuate emotional expression, or interrupt ... patterns of aversive, non-rewarding thought and emotion' (p. 819). However, without proper experimental control for such 'endogenous attempts', it is difficult to accept such an interpretation.

The neural networks implicated in mood disorders are likely to involve the midbrain as well as the limbic and prefrontal areas. Both major depressive disorder and bipolar affective disorder patients have demonstrated abnormally increased metabolism in the left thalamus. On the other hand, metabolism in the caudate is abnormally decreased in patients with major depressive disorder (Baxter et al, 1985). Drevets et al (1992) have proposed a limbic-cortical-striatal-pallidalthalamic circuit (Table 1). 
Table 1 Activity in the cortical-limbic-thalamic-striatal circuit in major depressive disorder and bipolar affective disorder

\begin{tabular}{|c|c|c|}
\hline \multicolumn{3}{|c|}{ Structure } \\
\hline Level & Increased activity ${ }^{1}$ & Decreased activity ${ }^{1}$ \\
\hline Cortex & $\begin{array}{l}\text { Insula, ventrolateral prefrontal cortex, } \\
\text { ventromedial prefrontal cortex, },^{2,3} \\
\text { subgenual anterior cingulate }\end{array}$ & $\begin{array}{l}\text { Dorsomedial and dorsolateral } \\
\text { prefrontal cortex }{ }^{2}\end{array}$ \\
\hline Midbrain & Thaslamus & \\
\hline Limbic system & Amygdala & \\
\hline Striatum & Ventral pallidum & Ventral striatum 2,4 \\
\hline $\begin{array}{l}\text { 1. After correcti } \\
\text { 2. Activity rever } \\
\text { 3. Ventromedial } \\
\text { 4. Activity in th } \\
\text { of caudate and }\end{array}$ & $\begin{array}{l}\text { volume changes. } \\
\text { mania. } \\
\text { ntal activity may be positively correlated } \\
\text { ate and putamen (ventral striatum is defi } \\
\text { en) may be negatively correlated with deg }\end{array}$ & $\begin{array}{l}\text { of anhedonia. } \\
\text { nucleus accumbens and ventral aspects } \\
\text { onia. }\end{array}$ \\
\hline
\end{tabular}

\section{Dynamic (task-related) studies}

Patients with major depressive disorder

Although resting-state studies have provided important clues about the functional neuroanatomy of mood disorder, they are at the mercy of independent metabolic and medication effects, and they are more sensitive to sample variation. Task-related designs have the potential to control for these independent variables: during the scans participants perform cognitive or motor tasks, or are exposed to sensory stimuli. Participants can therefore act as their own controls, as well as being compared with healthy volunteers.

Again, the medial prefrontal cortex has been a focus of much research, and cognitive tasks have been used in an attempt to demonstrate deficits. When compared with normal controls, patients with major depressive disorder have demonstrated reduced activation in the right mid-prefrontal cortex and the anterior cingulate cortex while performing tasks that tap into the processes of selective attention, cognitive interference and working memory (such as the continuous performance task, Stroop test and Tower of London task).

This is tentative support for the hypofrontality hypothesis of depression, but the demonstrated deficits are not specific for depression (similar deficits have been found in schizophrenia), and the extent to which cognitive paradigms can be used to explore the neural correlates of depressive symptoms is debatable. Furthermore, the cognitive effort may interrupt a normally high restingstate activity in the medial prefrontal area.

However, the hypofrontality hypothesis is further supported by a study demonstrating muted medial prefrontal activity in response to performance feedback in individuals in a depressed state carrying out a cognitive task (Elliott et al, 1998): during the Tower of London task a deficient neural response to positive or negative feedback was demonstrated in the medial caudate and ventromedial prefrontal cortex. According to Davidson et al (2002) the abnormality of prefrontal function in major depressive disorder may lead to deficient overriding of automatic processes that might involve the perseveration of negative affect and dysfunctional attitudes.

The functional significance of the prefrontal cortex partly relates to its participation in the modulation of responses to aversive and appetitive stimuli (Rolls, 1999). The medial prefrontal cortex probably plays a crucial role in the 'reward-related system': mesolimbic dopaminergic neurons project from the ventral tegmentum into the medial prefrontal cortex, amygdala and ventral striatum (Drevets et al, 2002).

In humans, dopamine release in the ventral striatum is tightly correlated with the euphoric response to dextroamphetamine, and medial prefrontal activity is normally positively related to rewarding experiences in healthy humans. So, suppressed medial prefrontal activity in depression could be associated with reduced activity in the striatum, because of less-active projections to this area. The perception of reward would thus be reduced, leading to the core depressive symptom of anhedonia (lack of pleasure).

However, sadness and depression may be associated with increased activity in certain parts of the medial prefrontal cortex - specifically the subgenual anterior cingulate. Perhaps this particular area has an inhibitory influence over the ventral striatum. A recent report (Keedwell et $a l, 2003)$ suggests that anhedonia is positively correlated with medial prefrontal activity and 
negatively correlated with ventral striatal activity in depressed people asked to recall happy memories (normally presumed to be a rewarding experience).

Putative disorders in the recognition of facial emotion have been examined in patients with major depressive disorder using functional neuroimaging. The neural responses of depressed and non-depressed individuals have been compared, to reveal biases. In one study fearful, neutral and happy facial expressions were presented to participants subliminally. The patients demonstrated increased left amygdala activation to all faces relative to normal controls but with a particularly strong response to fearful faces. This exaggerated amygdala activity subsided with successful treatment, so that the difference between patients and controls disappeared (Sheline et al, 2001). However, Drevets et al (1992) have suggested that increased left amygdala activation could be a trait marker of depression: they found that the elevated activity in this structure persisted after recovery from 'familial pure depressive disease'.

In support of the trait marker hypothesis for the amygdala, Hariri et al (2002) have found that serotonin transporter genetic variation could modulate the amygdala response to emotional faces. Thus, the evidence is converging on the idea that increased amygdala activation could represent a possible biological substrate for the cognitive bias towards aversive or emotionally arousing information in patients with depressive disorders. Drevets (2001) has suggested that increased amygdala activation might facilitate the emergence of depressive ruminations by increasing access to emotionally negative memories. A recent study (Siegle et al, 2002) showed that amygdala activation in response to negative words lasted for a significantly longer time in people currently experiencing episodes of depression than in normal controls. No such difference was demonstrated for neutral or positive words. Therefore the time course, rather than the intensity, of amygdala activation may mediate a perceptual bias toward stimuli with negative emotional meanings in patients during depressive episodes.

The extent to which the neural correlates of depressive brain activity in patients with major depressive disorder represent state or trait markers remains to be clarified.

Depressive episodes in bipolar affective disorder

Relatively few studies have examined the neural correlates of executive function and emotion processing in symptomatic patients with bipolar affective disorder. In patients with bipolar disorder experiencing episodes of depression, executive task performance and at-rest studies have demonstrated reduced metabolism in dorsolateral and dorsomedial prefrontal cortical regions but increased metabolism within the right amygdala, compared with healthy volunteers (Ketter et al, 2001). Reduced prefrontal and caudate metabolism to aversive stimulation has been demonstrated. The picture is therefore similar to that seen in people with major depressive disorder during episodes of depression.

\section{Manic states}

Understandably, the scanning of people in manic states represents an immense challenge for researchers because of hyperactive behaviour. Attempts have been made to compare brain activation changes during manic and depressive phases. In the manic phase, patients show increased activity in the left lateral prefrontal cortex when compared with the depressive phase (Baxter et al, 1989). In contrast, there may also be reductions in activity in the more medial prefrontal (orbitofrontal) cortical areas in mania compared with the euthymic state (Blumberg et al, 1999), suggesting a reciprocal relationship between medial and lateral prefrontal areas in mania. This would be consistent with the similar prefrontal dissociation seen for happy and sad moods and reward or punishment in healthy controls (see above). The findings are also consistent with the pattern of increased subgenual cingulate activity and reduced lateral prefrontal activity demonstrated in depression (above). In terms of the experience of pleasure, depression is typically the polar opposite to mania. Therefore, a reversal of the dissociated prefrontal activity might be expected.

However, when patients in the manic phase were compared with healthy controls in one study, increased activity was noted in the subgenual cingulate, similar to the picture in depression. Increased activity in the ventral striatum, which is implicated in the processing of reward, has been associated with the manic phase (Drevets et al, 1995; Blumberg et al, 2000), and this would not be surprising given that mania is often a subjectively pleasant experience.

As with major depressive disorder, there are contradictory findings for the role of the medial prefrontal area, and evidence is lacking that might help us to decide if the neural correlates of bipolar affective disorder represent state or trait features. A few studies have indicated a reduced extent of 
functional neuroanatomical abnormalities in euthymic bipolar affective disorder patients compared with symptomatic patients during executive task performance (Baxter et al, 1989; Blumberg et al, 1999, 2000).

The effect of psychotropic medication on the functional neuroanatomy of patients with mood disorders is largely unknown, although neuroleptic medication levels have been positively correlated with prefrontal cortical activation during decision-making in mania (Rubinsztein et al, 2001).

\section{Summary and future directions}

We have considered the neuropsychological, neuropathological and neuroimaging studies that have helped to map the brain regions whose abnormal structure or functioning have been associated with major depressive disorder and bipolar affective disorder. The prefrontal and anterior cingulate cortical areas are important, as are subcortical structures such as the amygdala, thalamus and striatum. There may be a dissociation of the direction of activity in some structures during manic as opposed to depressive episodes in bipolar affective disorder. There are still some inconsistencies between studies, owing to differences in methodologies and variability in findings.

We cannot specify whether the abnormalities of these structures are state or trait effects because longitudinal studies examining at-risk individuals are yet to be performed. Studies demonstrating neurocognitive abnormalities during euthymic episodes suggest that there are trait brain abnormalities associated with mood disorders. However, the findings are controversial because of the problem of 'residual symptoms' and it is not possible to determine whether these effects are primary, a consequence of the acute illness or a consequence of treatment.

Mapping the neuroanatomy of major depressive disorder and bipolar affective disorder helps to explain only one aspect of their pathogenesis. There is still much to learn about how cognitive and autonomic processes relate to one another via limbic structures. Neurochemical studies reveal highly complex interactions between amino acids (glutamate, gamma-aminobutyric acid, aspartate), monoamines (noradrenalin, acetylcholine, dopamine, serotonin), endorphins, glucocorticoids and corresponding receptors. A detailed consideration of these processes is beyond the scope of this article and can be found in reviews elsewhere (e.g. Pariante \& Miller, 2001).
We will ultimately gain a greater understanding of mood disorders by combining functional neuroanatomical and neurochemical approaches. Promising studies have examined the effects of lithium or antidepressants on brain structure, neurotransmitter function, and choline and myoinositol containing compounds (components of membrane phospholipids and cellular second messenger cycles). Macro and micro methods of investigation can both be used within the same paradigm to increase knowledge of neurochemical mediation: a neuroimaging study can employ participants with experimentally induced brain serotonin depletion, for example.

The development of imaging technologies has far outpaced their use in affective disorders. The simultaneous development of more powerful scanners, new neurochemical ligands, improved image generation and improved processing makes the future for understanding the neural mechanisms of mood disorders very promising.

\section{References}

Abercrombie, H. C., Schaefer, S. M., Larson, C. L., et al (1998) Metabolic rate in the right amygdala predicts negative affect in depressed patients. NeuroReport, 9, 3301-3307.

Attwell, D. \& Iadecola, C. (2002) The neural basis of functional brain imaging signals. Trends in Neurosciences, 25, 621-625.

Baumann, B., Danos, P., Krell, D., et al (1999) Reduced volume of limbic system-affiliated basal ganglia in mood disorders: preliminary data from a post mortem study. Journal of Neuropsychiatry and Clinical Neuroscience, 11, 71-78.

Baxter, L. R., Phelps, M. E., Mazziotta, J. C., et al (1985) Cerebral metabolic rates for glucose in mood disorders. Studies with positron emission tomography and fluorodeoxyglucose F18. Archives of General Psychiatry, 42, 441447.

—, Schwartz, J. M., Phelps, M. E., et al (1989) Reduction of prefrontal cortex glucose metabolism common to three types of depression. Archives of General Psychiatry, 46, 243-250

Bazin, N., Perruchet, P., DeBonis, M., et al (1994) The dissociation of explicit and implicit memory in depressed patients. Psychological Medicine, 24, 239-245.

Beyer, J. L. \& Krishnan, K. R. R. (2002) Volumetric brain imaging findings in mood disorders. Bipolar Disorders, 4, 89-104.

Blumberg, H. P., Eldelberg, D., Koscis, J. H., et al (1999) Rostral and orbital prefrontal cortex dysfunction in the manic state of bipolar disorder. American Journal of Psychiatry, 156, 1986-1988.

-, Stern, E., Martinez, D., et al (2000) Increased anterior cingulate and caudate activity in bipolar mania. Biological Psychiatry, 48, 1945-1952.

Blumer, D. \& Benson, D. F. (1975) Personality changes with frontal and temporal lesions. In Psychiatric Aspects of Neurologic Disease (eds D. F. Benson \& D. Blumer), pp. 155-157. New York: Grune \& Stratton.

Bowley, M. P., Drevets, W. C., Ongur, D., et al (2002) Low glial numbers in the amygdala in major depressive disorder. Biological Psychiatry, 52, 404-412.

Cavanagh, J. T. O., van Beck, M., Muir, W., et al (2002) Case-control study of neurocognitive function in euthymic patients with bipolar disorder: an association with mania. British Journal of Psychiatry, 180, 320-326. 
Clark, L., Iverson, S. D. \& Goodwin, G. M. (2002) Sustained attention deficit in bipolar disorder. British Journal of Psychiatry, 180, 313-319.

Cooley, E. L. \& Nowicki Jr, S. (1989) Discrimination of facial expressions of emotion by depressed subjects. Genetic, Social, and General Psychology Monographs, 115, 449-465.

Cotter, D., Mackay, D., Landau, S., et al (2001) Reduced glial cell density and neuronal size in the anterior cingulate cortex in major depressive disorder. Archives of General Psychiatry, 58, 545-553.

Damasio, H., Grabowski, T., Frank, R., et al (1994) The return of Phineas Gage: the skull of famous patient yields clues about the brain. Science, 264, 1102-1105.

Danion, J. M., Willard-Schroeder, D., Zimmermann, M. A., et al (1991) Explicit memory and repetition priming in depression. Preliminary findings. Archives of General Psychiatry, 48, 707-711.

Davidson, R. J., Pizzagalli, D., Nitschke, J. B., et al (2002) Depression: perspectives from affective neuroscience. Annual Review of Psychology, 53, 545-574.

Drevets, W. C. (2000) Neuroimaging studies of mood disorders. Biological Psychiatry, 48, 813-829.

- (2001) Neuroimaging and neuropathological studies of depression: implications for the cognitive-emotional features of mood disorders. Current Opinion in Neurobiology, 11, 240-249.

- Videen, T. O., Price, J. L., et al (1992) A functional anatomical study of unipolar depression. Journal of Neuroscience, 12, 3628-3641.

- Price, J. L., Videen, T. O., et al (1995) Metabolic abnormalities in the subgenual prefrontal cortex and ventral striatum in mood disorders. Neuroscience Abstracts, 21, 260.

—, - Simpson, J. R., et al (1997) Subgenual prefrontal cortex abnormalities in mood disorders. Nature, 386, 824827.

- - - Bardgett, M. E., et al (2002) Glucose metabolism in the amygdala in depression. Relationship to diagnostic subtype and plasma cortisol levels. Pharmacology, Biochemistry and Behavior, 71, 431-447.

Elliott, R., Sahakian, B. J., Michael, A., et al (1998) Abnormal neural response to feedback on planning and guessing tasks in patients with unipolar depression. Psychological Medicine, 28, 559-571.

Ferrier, I. N., Stanton, B. R., Kelly, T. P., et al (1999) Neuropsychological function in euthymic patients with bipolar disorder. British Journal of Psychiatry, 175, 246251

Gur, R. C., Erwin, R. J., Gur, R. E., et al (1992) Facial emotion discrimination. II: Behavioral findings in depression. Psychiatry Research, 42, 241-251.

Hale III, W. W. (1998) Judgment of facial expressions and depression persistence. Psychiatry Research, 80, 265-274.

Hariri, A. R., Mattay, V. S. \& Tessitore III, A. (2002) Serotonin transporter genetic variation and the response of the human amygdala. Science, 297, 400-403.

Keedwell, P. A., Andrew, C., Williams, S. C. R., et al (2003) The neural correlates of depression (abstract). Biological Psychiatry, 53, 171s.

Ketter, T. A., Kimbrell, T. A., George, M. S., et al (2001) Effects of mood and subtype on cerebral glucose metabolism in treatment-resistant bipolar disorder. Biological Psychiatry, 49, 97-109.

Logothetis, N. K., Pauls, J., Augath, M., et al (2001) Neurophysiological investigation of the basis of the fMRI signal. Nature, 412, 150-157.

Manji, H. K., Moore, G. J., Rajkowska, G., et al (2000) Neuroplasticity and cellular resilience in mood disorders. Molecular Psychiatry, 5, 578-593.

Matthews, G. R. \& Antes, J. R. (1992) Visual attention and depression: cognitive biases in the eye fixation of the dysphoric and non-depressed. Cognitive Therapy and Research, 16, 359-371.

Mayberg, H. S. (2000) Depression. In Brain Mapping: The Disorders (eds J. C. Mazziotta, A. W. Toga \& R. S. J Frackowiak). London: Academic Press.
-, Liotti, M., Brannan, S. K., et al (1999) Reciprocal limbiccortical function and negative mood. Converging PET findings in depression and normal sadness. American Journal of Psychiatry, 156, 675-682.

_- Brannan, S. K., Tekell, J. L., et al (2000) Regional metabolic effects of fluoxetine in major depression: serial changes and relationship to clinical response. Biological Psychiatry, $48,830-843$

Mikhailova, E. S., Vladimirova, T. V., Iznak, A. F., et al (1996) Abnormal recognition of facial expression of emotions in depressed patients with major depression disorder and schizotypal personality disorder. Biological Psychiatry, 40 , 697-705.

Northoff, G., Richter, A., Gessner, M., et al (1999) Functional dissociation between medial and lateral prefrontal cortical spatiotemporal activation in negative and positive emotions. A combined fMRI/MEG study. Cerebral Cortex, 10, 93.

Ongur, D., Drevets, W. C. \& Price, J. L. (1998) Glial reduction in the subgenual prefrontal cortex in mood disorders. Proceedings of the National Academy of Sciences of the USA, 95, 13290-13295.

Pardo, J. V., Pardo, P. J. \& Raichle, M. E. (1993) Neural correlates of self-induced dysphoria. American Journal of Psychiatry, 150, 713-719.

Pariante, C. M. \& Miller, A. H. (2001) Glucocorticoid receptors in major depression: relevance to pathophysiology and treatment. Biological Psychiatry, 49, 391-404.

Perrett, D. I., Rolls, E. T. \& Caan, W. (1982) Visual neurones responsive to faces in the monkey temporal cortex. Experimental Brain Research, 47, 329-342.

Rajkowska, G., Halaris, A. \& Selemon, L. D. (2001) Reductions in neuronal and glial density characterize the dorsolateral prefrontal cortex in bipolar disorder. Biological Psychiatry, 49, 741-752.

Rolls, E. T. (1999) The functions of orbitofrontal cortex. Neurocase, 5, 301-312.

Rubinow, D. R. \& Post, R. M. (1992) Impaired recognition of affect in facial expression in depressed patients. Biological Psychiatry, 31, 947-953.

Rubinsztein, J. S., Fletcher, P. C., Rogers, R. D., et al (2001) Decision-making in mania: a PET study. Brain, 124, 25502563.

Sheline, Y. I., Barch, D. M., Donnelly, J. M., et al (2001) Increased amygdala response to masked emotional faces in depressed subjects resolves with antidepressant treatment: an fMRI study. Biological Psychiatry, 50, 651-658.

Siegle, G. J., Steinhauer, S. R., Thase, M. E., et al (2002) Can't shake that feeling: event-related fMRI assessment of sustained amygdala activity in response to emotional information in depressed individuals. Biological Psychiatry, 51, 693-707.

Suslow, T., Junghanns, K. \& Arolt, V. (2001) Detection of facial expressions of emotions in depression. Perceptual and Motor Skills, 92, 857-868.

Talairach, J. \& Tournoux, P. (1988) Co-planar Stereotactic Atlas of the Human Brain. Stuttgart: Thieme.

Veiel, H. O. F. (1997) A preliminary profile of neuropsychological deficits associated with major depression. Journal of Clinical and Experimental Neuropsychology, 19, 587-603.

Watkins, P. C., Vache, K., Verney, S. P., et al (1996) Unconscious mood-congruent memory bias in depression. Journal of Abnormal Psychology, 105, 34-41.

\section{Multiple choice questions}

1 The following is true about functional imaging:

a PET scans have better temporal resolution than fMRI

b fMRI has better spatial resolution than PET

c PET can measure glucose metabolism

d there is a well-established linear relationship between the BOLD response and neural excitation. 
2 Established components of the 'reward system' include:

a ventral tegmentum

b ventral striatum

c medial prefrontal cortex

d cerebellum.

3 With regard to bipolar affective disorder:

a mania and depression may correlate with increased activation in distinct parts of the prefrontal cortex

$\mathrm{b}$ bipolar depression is associated with increased subgenual cortex activation

c lithium may provide protection against some structural brain changes

$\mathrm{d}$ the amygdala is not affected.

4 With regard to major depressive disorder:

a the hippocampus may be reduced in volume

b cognitive deficits are confined to the frontal lobe

c antidepressant treatment has been shown to reverse subgenual changes in neural activation

$\mathrm{d}$ there may be a negative bias in the recognition of facial expressions related to altered amygdala function.
5 In the neuropathology of mood disorder:

a a reduction in grey matter volume has been demonstrated in the orbitofrontal cortex and the amygdala

$b$ changes in grey matter volume are due to a reduction in the numbers of neurons

c the size of a brain structure gives a good indication of its function

d patients with unmedicated bipolar affective disorder have been shown to have reduced grey matter volume in the amygdala.

\section{MCQ answers}

$\begin{array}{llllllllll}1 & & 2 & & 3 & & 4 & & 5 & \\ \text { a } & \text { F } & \text { a } & \text { T } & \text { a } & \text { T } & \text { a } & \text { T } & \text { a } & \text { T } \\ \text { b } & \text { T } & \text { b } & \text { T } & \text { b } & \text { T } & \text { b } & \text { F } & \text { b } & \text { F } \\ \text { c } & \text { T } & \text { c } & \text { T } & \text { c } & \text { T } & \text { c } & \text { T } & \text { c } & \text { F } \\ \text { d } & \text { F } & \text { d } & \text { F } & \text { d } & \text { F } & \text { d } & \text { T } & \text { d } & \text { T }\end{array}$

\section{Child and Adolescent Mental Health Services:} An Operational Handbook

\section{Edited by Greg Richardson and lan Partridge}

Now is a crucial time for child and adolescent mental health services (CAMHS). They have to integrate with all the other current children's initiatives and develop in line with the Children's National Service Framework to ensure the mental health needs of all children are met.

This book tells you how to do just that. It explains in straightforward operational terms how services can be delivered in ways in which children, families and other agencies can understand, that are as local to the child and family as possible, that are helpful to educational, social, voluntary and other partner agencies and that allow clear commissioning processes. The operation of each of the four tiers of service provision is clearly described with specific examples at each tier. Those providing, working in, working with and commissioning CAMHS will find it an indispensable guide.

- Straightforward, 'how to' guide.

- Focus on inter-agency working within each tier of service.

- Practical examples of service provision.

- Geared towards all professionals working with CAMHS

September 2003, 214pp, paperback, ISBN 190124296 X, Price $£ 25.00$

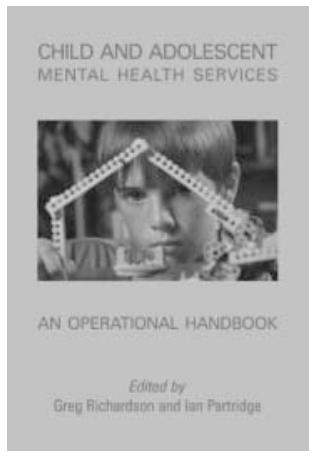

